\title{
Foreword to the Special Issue on Optical Technologies for Biomedical Applications
}

We are pleased to present the special issue of JBPE, which focuses on optical technologies for different biomedical applications. The issue contains selected papers presented at Saratov Fall Meeting 2016 (SFM-16) - International Symposium on Optics and Biophotonics - IV (September 27-30, 2016, Saratov, Russia) and includes eight representative papers that well characterize the major topics of SFM-16.

Invited paper of E. Zherebtsov and co-authors is based on a plenary lecture presented by Prof. A. Dunaev during SFM-16. This paper describes current state of the optical non-invasive diagnostics, especially methodological and metrological provision for this technology. The authors focus on laser Doppler flowmetry and fluorescence spectroscopy.

Invited paper of $\mathbf{L}$. Oliveira and co-authors is related to the study of wavelength dependence of the refractive index of healthy and pathological human colorectal mucosa. Authors underline that refractive index, which can be measured directly, is very significant for the improvement or development of optical technologies in clinical practice. The presented results have demonstrated that it is possible to discriminate between healthy and pathological tissues from refractive index measurements.

Investigations of refractive index dispersion, permittivity and absorption coefficient of human nails in THz frequency range are presented in paper of $\mathbf{V}$. Guseva and co-authors. These results may be used for development of non-invasive technique of human pathology monitoring using a nail as a reference sample in reflection mode of $\mathrm{THz}$ time-domain spectroscopy.

The main blood protein albumin attracts much attention of researchers. E. Nepomnyashchaya and co-authors have investigated the albumin-fullerenol interaction by laser correlation spectroscopy. Authors have suggested a new algorithm for the solution of the inverse problem allowing one to analyze sizes of nanoparticles in polydisperse solutions.

Deficiency or over exposure to iron of the living organism can cause various diseases ranging from anemia to iron overload, and possibly to neurodegenerative diseases. Therefore, it is of undoubted interest to study the molecular dynamics of the processes occurring in aqueous solutions of serum albumin under the influence of iron ions. V. Gibizova and co-authors have investigated the molecular mobility of the albumin molecules in pure aqueous solution and at the addition of ferric chloride III by light scattering method.

Photosensitizing properties of novel supramolecular systems based on chlorin e6 are discussed in paper of I. Klimenko and A. Lobanov. The influence of various excipients on spectral characteristic of these systems as well as the influence of intermolecular interactions on aggregation behavior of chlorin e6 in solutions have been studied.

The use of nanostructures in therapy is an actual problem in the medicine. The bactericidal activity of nanocomposites prepared on the basis of natural glauconite matrix and antibiotics against Staphylococcus aureus has been demonstrated by S. Venig and co-authors. The authors note the prospects for use of glauconite composites in human and veterinary medicine.

For the past few years, there has been a rapid development of nanovaccine design, which are on use to solve relevant problems of prevention and treatment of human and animal diseases. P. Mezhenny and co-authors present the study of immunogenic properties of transmissible gastroenteritis virus antigen conjugated with gold and selenium nanoparticles.

In overall, papers collected in this special issue demonstrate well the exciting potential of optical technologies for biomedical studies and applications aiming medical diagnostics and treatment. 
INTRODUCTION

Special issue Editors:

\section{Elina A. Genina, Alexey N. Bashkatov,}

Optics and Biophotonics Department, Research and Educational Institute of Optics and Biophotonics of the Saratov National Research State University; Biophotonics Laboratory of the Tomsk National Research State University, Russia

\section{Valery V. Tuchin,}

Optics and Biophotonics Department, Research and Educational Institute of Optics and Biophotonics of the Saratov National Research State University; Institute of Precision Mechanics and Control RAS; Biophotonics Laboratory of the Tomsk National Research State University; Samara National Research University, Russia 